\section{Intratest Scatter}

Sandra Banks ${ }^{1}$ and Michael Franzen ${ }^{2}$

${ }^{1}$ Department of Psychiatry, Allegheny General Hospital, Pittsburgh, PA, USA

${ }^{2}$ Allegheny Neuropsychiatric Institute, Pittsburgh, PA, USA

\section{Synonyms}

Intratest variability

\section{Definition}

Intratest scatter is a measure of the variability of item scores within a test. High variability in responses will lead to a wide scattering of scores around the mean score on the test. Low variability will lead to a minimal scattering of scores around the mean score. The degree of scatter needed to be considered abnormal is a function of the correlation within the test and the reliability of the individual scores. Scatter in tests that do not demonstrate a high correlation may not reflect pathology. Scatter among scores within a test that demonstrate low reliability may not reflect pathology.

Specific patterns of scores within tests may assist with diagnosis. For example, if an individual's performance on the continuous performance test is inconsistent in terms of reaction times in response to test stimuli across the test, this will result in an intratest scatter suggestive of poor sustained attention and vigilance.

\section{Cross-References}

Test Interpretations 